\title{
Using IP as Transport Technology in Third Generation and Beyond Radio Access Networks
}

\author{
Attila Báder ${ }^{1}$, Lars Westberg ${ }^{2}$, and Georgios Karagiannis ${ }^{3}$ \\ ${ }^{1}$ Traffic Lab, Ericsson Research, P.O. Box 107, Budapest, Hungary, H-1300 \\ ${ }^{2}$ Ericsson Research, Torshamnsgatan 23, SE-16480, Stockholm, Sweden \\ \{attila.bader, lars.westberg\} @ericsson.com \\ ${ }^{3}$ University of Twente, P.O. Box 217, 7500 AE Enschede, the Netherlands \\ g.karagiannis@utwente.nl
}

\begin{abstract}
This paper discusses the motivation for developing a new QoS signaling protocol for IP-based Radio Access Networks. It describes the main characteristics of these networks and the special requirements imposed by these characteristics on QoS signaling solutions.
\end{abstract}

\section{Introducing IP Transport in Radio Access Networks: Motivation}

Due to its flexibility and cost efficiency, IP transport is gradually introduced in different parts of third generation and beyond cellular networks, including their Radio Access Network (RAN).

The discussion related to the introduction of the IP technology in radio access networks is valid for all types of cellular systems, i.e., GPRS and IMT2000 (e.g. CDMA2000 and UMTS). For reasons of clarity, we will only focus on the Universal Mobile Telecommunication System (UMTS). Third generation and beyond cellular networks consist of access and core networks. In case of the UMTS Terrestrial RAN (UTRAN), see Figure 1, the boundaries are the radio base stations (i.e., Node Bs) and the Radio Network Controllers (RNCs) [1]. The Node B provides the radio channel coding/decoding and transmission/reception function for User Equipments (UE) in its coverage area called cell. The RNC controls the radio resources (the radio channels and the connections) of a number of Node Bs. The RNC is also responsible for controlling the soft handover combining and splitting between streams from different Node Bs belonging to the same User Equipment. Furthermore, the RNC is responsible for the allocation of transport resources within the RAN. The Mobile Switching Centre (MSC) and Media Gateway (MGW) are used for circuit-switched services towards other User Equipments and external circuit-switched networks. The SGSN and GGSN entities provide support for packet switched services towards User Equipments, including mobility management, access control and control of packet data protocol contexts. In addition, the GGSN provides inter-working with external packet-switched networks such as the public Internet.

The IP technology can be considered as an open transport architecture that can run on top of many various link layer techniques, such as Ethernet, SDH, ATM. The 


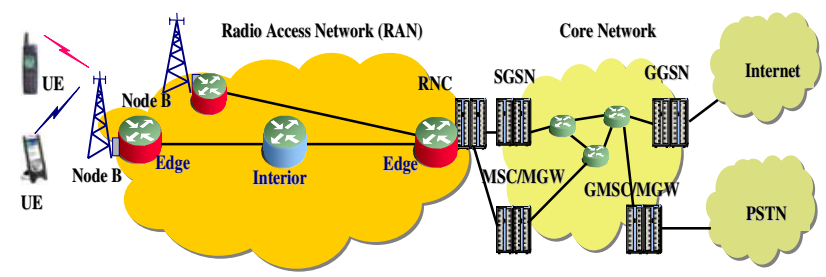

Fig. 1. Third generation cellular network scenario

choice of the link layer techniques is completely transparent to the higher layers, making it possible to use several solutions at the same time. Ethernet for example can be cost efficient choice in a metropolitan area.

Considering that IP is already used in the transport part of the UMTS core network, if IP will also be used in the transport part of the UTRAN, then one single network management system can be used for both UMTS core and radio access network (full IP network). Due to the auto-configuration capabilities of IP updating the network to higher traffic volume or wider coverage is simpler than e.g. in case of ATM network.

Using IP-based transport within the UTRAN, gives operators an opportunity to upgrade their transport network to a packet-based one. When compared with a traditional STM-based system, the gain is seen in the statistical multiplexing of traffic. As a tradeoff, an efficient QoS solution has to be developed for IP based RAN.

\section{Characteristics of RANs and Imposed QoS Requirements}

The characteristics of the RAN impose special requirements on the resource reservation protocol that is used within the RAN. The main characteristics of radio access networks are the following:

- Very often the transmission in a RAN contains a relatively high volume of expensive leased lines [3].

- The fact that Node Bs are spread over a large geographic area and in general far away from the backbone typically results in high cost of the transmission links. Therefore, efficient bandwidth utilization in UTRAN is essential.

- A majority of the traffic transported on the transmission links of UTRAN are radio frames, which are short data segments that are transmitted by the Node B on a given radio channel and given time slots [4,5]. The traffic is, therefore, very sensitive both for delay and jitter. The QoS requirement is especially strict for voice traffic that is usually dominating in the networks.

- Due to the mobility, and in order to efficiently utilize the radio resources, the UTRAN supports frequent handover procedures between radio channels and Node Bs. Supporting soft handover (between Node Bs controlled by the same $\mathrm{RNC}$ ) in $3 \mathrm{G}$ network releases, puts the most demanding requirements on the resource reservation protocol.

- In the early phase of $3 \mathrm{G}$ networks the voice traffic dominates in the network. By introducing HSDPA (High Speed Downlink Packet Access) service, the band- 
width demand increases rapidly and the UTRAN transport network may become the main bottleneck of the UMTS network.

- A potential UTRAN network is large, consisting of several hundred Node Bs that are generating an increasing number of traffic volume over the lifetime of the network. Even if a single Node B generates a modest traffic volume, the total amount of flows for radio frame transport in the radio access network is significantly large.

Though several other QoS solutions can be applied for IP-based RAN, signaled QoS is fairly the most effective in such a dynamic environment. The special characteristics of RANs arises the need to identify the requirements of a resource reservation signaling protocol that can be used within IP based UTRAN:

- The resource reservation between the edges of the RAN, see Figure 1, is imposed by radio specific functions, and not by the end-to-end resource reservation signaling. Therefore, the resource reservation in the UTRAN may be different from the QoS solutions in the other part of the network. If the resource reservation in the UTRAN is part of an end-to-end resource reservation protocol, the protocol should support edge-to-edge reservations as well. Support is also necessary for bi-directional unicast reservations for delay-sensitive traffic, due to the nature of the radio frame traffic flows $[4,5]$.

- The peak bit rate of the multi-rate radio channels is selected on-demand [6]. To efficiently utilize the bandwidth of the expensive transmission links used for radio frame transport, over-provisioning is in general not feasible and, therefore, the protocol would be used for on-demand reservation.

- At each handover event, the resource reservation may be reinitiated. As a consequence of the frequent handover, the resource reservation protocol will be used very frequently and needs to be fast.

- Due to the large number of flows and high traffic that have to be supported within the UTRAN, the protocol needs to be scalable.

The NSIS working group in IETF develops a QoS signaling protocol that takes into account the above requirements [2]. The Resource Management in Diffserv Quality of Service Model, whichcan be used within the NSIS protocol suite, is especially suitable for large-scale RAN applications [7].

\section{References}

1. 3GPP TS 25.401: "UTRAN Overall Description".

2. Brunner, M., "Requirements for Signaling Protocols" IETF Request for Comments 3726.

3. Bjarhov, M., Friberg, C., "GSM network solutions for new-growth markets", Ericsson Review, Issue nr. 01, 2004.

4. 3GPP TS 25.104: "UTRA (BS) FDD; Radio transmission and Reception".

5. 3GPP TS 25.105: "UTRA (BS) TDD; Radio transmission and Reception".

6. 3GPP TS 26.093: "Source Controlled Rate operation ".

7. A. Bader, L. Westberg, G. Karagiannis, C. Kappler, T. Phelan, H. Tschofenig: "RMDQOSM - The Resource Management in Diffserv Quality of Service Model. " Internet draft, work in progress, Feb. 2005. 